\title{
SZEGÖ'S CONJECTURE ON LEBESGUE CONSTANTS FOR LEGENDRE SERIES
}

\author{
C. K. QU AND R. WONG
}

In 1926, Szegō conjectured that the Lebesgue constants for Legendre series form a monotonically increasing sequence. In this paper, we prove that his conjecture is true. Our method is based on an asymptotic expansion together with an explicit error bound, and makes use of some recent results of Baratella and Gatteschi concerning uniform asymptotic approximations of the Jacobi polynomials.

1. Introduction. The Lebesgue constants for classical Fourier series are defined by

$$
\rho_{n}=\frac{1}{\pi} \int_{0}^{\pi} \frac{|\sin (n+1 / 2) t|}{\sin (t / 2)} d t, \quad n=1,2,3, \ldots ;
$$

see [18, p. 172]. Fejer [4] was the first to show that

$$
\rho_{n}=\frac{4}{\pi^{2}} \log n+c_{0}+\frac{c_{1}}{n}+\frac{\alpha(n)}{n^{2}},
$$

where $c_{0}$ and $c_{1}$ are constants and $\alpha(n)$ is bounded for all $n$. From (1.2), he deduced that

$$
\rho_{n+1}-\rho_{n}>0
$$

for large $n$. He further conjectured that (1.3) holds for all $n \geq 1$, a conjecture later proved by Gronwall [7]. Gronwall's result was considerably improved by Szegö [12], who showed that the sequence of differences of the Lebesgue constants $\rho_{n}$ is in fact completely monotonic, i.e., $\Delta \rho_{n}=\rho_{n+1}-\rho_{n}>0$ and $(-1)^{r-1} \Delta^{r} \rho_{n}>0$ for $r=2,3, \ldots$

In exactly the same manner, one can investigate the properties of the Lebesgue constants

$$
\text { (1.4) } \begin{aligned}
L_{n} & =\frac{n+1}{2} \int_{-1}^{1}\left|P_{n}^{(1,0)}(x)\right| d x \\
& =(n+1) \int_{0}^{\pi} \sin \frac{\theta}{2} \cos \frac{\theta}{2}\left|P_{n}^{(1,0)}(\cos \theta)\right| d \theta, \quad n=1,2, \ldots,
\end{aligned}
$$

for Legendre series at $x=1$, where $P_{n}^{(1,0)}(x)$ is the Jacobi polynomial with $\alpha=1$ and $\beta=0$. The asymptotic formula

$$
L_{n}=\frac{2^{3 / 2}}{\sqrt{\pi}} n^{1 / 2}+o\left(n^{1 / 2}\right), \quad n \rightarrow \infty
$$


was first given by Gronwall [8] and later, by Szegö [14, 15] with simpler proofs. In 1926, Szegö conjectured that this sequence is monotonically increasing, i.e.,

$$
L_{n+1}-L_{n}>0
$$

for all $n \geq 1$; see footnote 6 in [13] and also the editor's comment on page 313 of [1]. In order to settle his conjecture for at least large values of $n$, Szegö posed to Lorch in the fifties the problem of obtaining more refined results than that given in (1.5). In [10], Lorch showed that

$$
L_{n}=\frac{2^{3 / 2}}{\sqrt{\pi}} n^{1 / 2}+B_{0}+O\left(n^{-1 / 2}\right),
$$

where $B_{0}$ is a constant. More explicitly, if $j_{\nu, k}$ denotes the $k$ th positive zero of the Bessel function $J_{\nu}(x)$, and if

$$
M_{k}=(-1)^{k} J_{0}\left(j_{1, k}\right),
$$

then

$$
B_{0}=1+2 \sum_{k=1}^{\infty}\left\{M_{k}-\frac{2^{3 / 2}}{\pi}\left[k^{1 / 2}-(k-1)^{1 / 2}\right]\right\},
$$

where the infinite series is absolutely convergent. A simple calculation reveals that the result in (1.7) is insufficient to determine the asymptotic monotonicity of the sequence $L_{n}$. Lorch thus proposed to one of us (Wong) in 1980 the problem of replacing the $O$-term in (1.7) by an explicitly determined expression plus terms of lower asymptotic order. The following result provides a solution to his problem, and is proved in [6]:

$$
L_{n}=\frac{2^{3 / 2}}{\sqrt{\pi}} n^{1 / 2}+B_{0}+\sqrt{\frac{2}{\pi}} n^{-1 / 2}+D_{0} n^{-1}+\varepsilon(n),
$$

where

$$
\begin{aligned}
D_{0}=\sum_{k=1}^{\infty}\left\{\hat{M}_{k}\right. & -\frac{2^{3 / 2}}{3}\left[k^{3 / 2}-(k-1)^{3 / 2}\right] \\
& \left.-2^{-3 / 2}\left[k^{1 / 2}-(k-1)^{1 / 2}\right]\right\}
\end{aligned}
$$

and

$$
\hat{M}_{k}=(-1)^{k+1}\left(j_{0, k}\right) J_{1}\left(j_{0, k}\right) .
$$

The remainder $\varepsilon(n)$ in $(1.10)$ satisfies

$$
\varepsilon(n)=O\left(n^{-3 / 2}\right), \quad \text { as } n \rightarrow \infty,
$$


and the infinite series in (1.11) is absolutely convergent. From (1.10), it follows immediately that $\left\{L_{n}\right\}$ is an asymptotically increasing sequence.

The purpose of this paper is to demonstrate that (1.6) holds for all $n \geq 1$, i.e., Szegö's conjecture is true. Our argument is based on the asymptotic representation (1.10) together with the improved numerical estimate

$$
|\varepsilon(n)| \leq \frac{15}{n^{3 / 2}} \text { for all } n \geq 49 .
$$

From (1.14), it will be proved that (1.6) holds for all $n \geq 49$. The first fifty $\rho_{n}$ can be calculated numerically, and their values are exhibited in the table in $\S 3$. An examination of these values shows that the sequence $\left\{L_{n}\right\}$ is indeed monotonically increasing.

To prove (1.14), we shall make use of some recent results of Baratella and Gatteschi [2] concerning asymptotic approximations of Jacobi polynomials and their zeros. Although these results are in a sense refinements of the asymptotic approximations obtained by Frenzen and Wong [5], they are of quite different nature from those given in [5]. Thus, in spite of the fact that the main strategy in this paper is similar to that employed by Frenzen and Wong [6], the detailed analysis here differs considerably from that given there.

The content of this paper is arranged as follows. In $\S 2$, we collect some of the known results to be used later in the paper. The main sketch of the argument is presented in $\S 3$. Many of the results in $\S 3$ are proved in subsequent sections.

2. Some preliminary results. In [5], Frenzen and Wong have derived the following asymptotic expansion for the Jacobi polynomial.

Theorem A. For $\alpha>-\frac{1}{2}, \alpha-\beta>-2 p$ and $\alpha+\beta \geq-1$, we have

$$
\begin{aligned}
(2.1)\left(\sin \frac{\theta}{2}\right)^{\alpha} & \left(\cos \frac{\theta}{2}\right)^{\beta} P_{n}^{(\alpha, \beta)}(\cos \theta) \\
& =\frac{\Gamma(n+\alpha+1)}{n !}\left(\frac{\theta}{\sin \theta}\right)^{1 / 2}\left[\sum_{l=0}^{p-1} A_{l}(\theta) \frac{J_{\alpha+l}(N \theta)}{N^{\alpha+l}}+\sigma_{p}\right],
\end{aligned}
$$

where

$$
N=n+\frac{1}{2}(\alpha+\beta+1)
$$

and

$$
\sigma_{p}=\theta^{\alpha} O\left(N^{-p}\right)
$$


the O-term being uniform with respect to $\theta \in[0, \pi-\varepsilon], \varepsilon>0$. The coefficients $A_{l}(\theta)$ are analytic functions in $0 \leq \theta \leq \pi-\varepsilon$, and are $O\left(\theta^{l}\right)$ in that interval. In particular, $A_{0}(\theta)=1$ and

$$
A_{1}(\theta)=\frac{1}{16}\left[\left(4 \alpha^{2}-1\right)\left(\frac{2}{\theta}-\cot \frac{\theta}{2}\right)+\left(4 \beta^{2}-1\right) \tan \frac{\theta}{2}\right] .
$$

It is this result that has led to the four-term asymptotic expansion of $L_{n}$ given in (1.10). Motivated by Theorem A, Baratella and Gatteschi [2] showed that $P_{n}^{(\alpha, \beta)}(\cos \theta)$ also has the Cherry-type approximation [3] given in Theorem B below, complete with an explicit error bound. Let

$$
f_{1}(\theta)=\left(1-4 \alpha^{2}\right)\left(\frac{2}{\theta}-\cot \frac{\theta}{2}\right)+\left(1-4 \beta^{2}\right) \tan \frac{\theta}{2}
$$

and

$$
f(\theta)=N \theta+\frac{1}{16 N} f_{1}(\theta)
$$

cf. (2.4). From the Maclaurin series expansion of $\tan (\theta / 2)$ and $(2 / \theta-$ $\cot (\theta / 2))$, it is easily seen that if $-\frac{1}{2} \leq \alpha, \beta \leq \frac{1}{2}$, then $f_{1}(\theta)$ is positive and increasing in $(0, \pi)$. Since $f(0)=0$ and

$$
f\left(\frac{\pi}{2}\right)=N \frac{\pi}{2}+\frac{1}{16 N} f_{1}\left(\frac{\pi}{2}\right)>\frac{\pi}{2} \quad \text { if } N>1,
$$

the equation $f(\theta)=\pi / 2$ has a unique root $\theta^{*}$ in $(0, \pi / 2)$.

THEOREM B. Let $-\frac{1}{2} \leq \alpha, \beta \leq \frac{1}{2}$ and let $\theta^{*}$ be the root of the transcendental equation $f(\theta)=\pi / 2$. Then

$$
\begin{aligned}
\text { (2.7) } & {\left[\frac{f(\theta)}{f^{\prime}(\theta)}\right]^{-1 / 2}\left(\sin \frac{\theta}{2}\right)^{\alpha+1 / 2}\left(\cos \frac{\theta}{2}\right)^{\beta+1 / 2} P_{n}^{(\alpha, \beta)}(\cos \theta) } \\
& =2^{-1 / 2} N^{-\alpha} \frac{\Gamma(n+\alpha+1)}{n !}\left[1+\frac{1}{32 N^{2}}\left(\frac{A}{3}+B\right)\right]^{-\alpha} J_{\alpha}[f(\theta)]-I,
\end{aligned}
$$

where $A=\left(1-4 \alpha^{2}\right), B=\left(1-4 \beta^{2}\right)$, and for $N \geq 5$

$$
|I| \leq\left\{\begin{array}{lr}
\theta^{\alpha} N^{-4}\left(\begin{array}{c}
n+\alpha \\
n
\end{array}\right)[0.00812 A+0.0828 B], & 0<\theta \leq \theta^{*}, \\
\theta^{1 / 2} N^{-\alpha-7 / 2}\left(\begin{array}{c}
n+\alpha \\
n
\end{array}\right)[0.0526 A+0.535 B], & \theta^{*} \leq \theta \leq \frac{\pi}{2}
\end{array}\right.
$$

As we shall see in this paper, it is this latter result which has led to the error estimate (1.14). For the Lebesgue constant (1.4), we need $\alpha=1$ and $\beta=0$, a case not included in Theorem B. Nevertheless, by a slight modification of the argument given in [2], we have the following corollaries. 
Corollary 1. Let $N=n+1 \geq 50$, and let

$$
f(\theta)=N \theta+\frac{1}{16 N}\left[\tan \frac{\theta}{2}-3\left(\frac{2}{\theta}-\cot \frac{\theta}{2}\right)\right] \equiv N \theta+\frac{1}{16 N} f_{1}(\theta) \text {, }
$$

and let $\theta^{*}$ be the root of the equation $f(\theta)=\pi / 2$. Then

$$
\begin{aligned}
& {\left[\frac{f(\theta)}{f^{\prime}(\theta)}\right]^{-1 / 2}\left(\sin \frac{\theta}{2}\right)^{3 / 2}\left(\cos \frac{\theta}{2}\right)^{1 / 2} P_{n}^{(1,0)}(\cos \theta)} \\
& \quad=2^{-1 / 2} J_{1}[f(\theta)]+I,
\end{aligned}
$$

where

$$
|I| \leq \begin{cases}0.0059 N^{-3} \theta, & 0<\theta \leq \theta^{*} \\ 0.1581 N^{-7 / 2} \theta^{1 / 2}, & \theta^{*} \leq \theta \leq \frac{\pi}{2}\end{cases}
$$

A precise estimate for the root $\theta^{*}$ can be obtained as follows. Since $f_{1}(\theta)$ is positive and $(1 / \theta) f_{1}(\theta)$ is strictly increasing in $(0, \pi / 2]$, we have

$$
N \theta \leq f(\theta) \leq N \theta+\frac{\theta}{2 N \pi}\left(1-\frac{3}{\pi}\right) .
$$

From this, it is easily verified that for $N \geq 50$,

$$
0.999997 \frac{\pi}{2 N}<\theta^{*}<\frac{\pi}{2 N} .
$$

In view of the well-known identity $[16, p .59]$

$$
P_{n}^{(1,0)}(\cos \theta)=(-1)^{n} P_{n}^{(0,1)}(-\cos \theta)=(-1)^{n} P_{n}^{(0,1)}(\cos (\pi-\theta))
$$

and the fact that the interval of integration in $(1.4)$ is $(0, \pi)$, we also need an asymptotic approximation for $P_{n}^{(0,1)}(\cos \theta)$.

Corollary $1^{\prime}$. Let $N=n+1 \geq 50$, let

$$
\begin{aligned}
\hat{f}(\theta) & =N \theta-\frac{1}{16 N}\left[3 \tan \frac{\theta}{2}-\left(\frac{2}{\theta}-\cot \frac{\theta}{2}\right)\right] \\
& =N \theta-\frac{1}{16 N} \hat{f}_{1}(\theta),
\end{aligned}
$$

and let $\hat{\theta}^{*}$ be the root of $\hat{f}(\theta)=\pi / 2$. Then

$$
\begin{gathered}
{\left[\frac{\hat{f}(\theta)}{\hat{f}^{\prime}(\theta)}\right]^{-1 / 2}\left(\sin \frac{\theta}{2}\right)^{1 / 2}\left(\cos \frac{\theta}{2}\right)^{3 / 2} P_{n}^{(0,1)}(\cos \theta)} \\
=2^{-1 / 2} J_{0}[\hat{f}(\theta)]+\hat{I},
\end{gathered}
$$


where

$$
|\hat{I}| \leq \begin{cases}0.0843 N^{-4}, & 0<\theta \leq \hat{\theta}^{*} \\ 0.5713 N^{-7 / 2} \theta^{1 / 2}, & \hat{\theta}^{*} \leq \theta \leq \frac{\pi}{2} .\end{cases}
$$

By using an argument similar to that for (2.13), it can be shown that

$$
\frac{\pi}{2 N}<\hat{\theta}^{*}<1.00004 \frac{\pi}{2 N}
$$

for $N \geq 50$.

The method used by Frenzen and Wong [6] consists of a subdivision of the interval of integration $(0, \pi)$ in $(1.4)$ at the zeros of the Jacobi polynomials and an application of the uniform asymptotic expansion given in Theorem A. In this paper, we shall approach the problem in a different manner. We shall first replace the Jacobi polynomial in (1.4) by its asymptotic approximation given in Corollary 1 , and then split the interval $(0, \pi / 2)$ at the roots $\tau_{k}$ of the equation $f(\theta)=j_{1, k}$, where $j_{1, k}$ is the $k$ th positive zero of $J_{1}(x)$.

LEMMA 1. Let $f(\theta)$ be the function defined by (2.9) and let $\tau_{k}$ denote the root of the equation $f(\theta)=j_{1, k}$. (a) If $\bar{n}=[n / 2]$ and $N=n+1 \geq 5$, then

$$
\tau_{\bar{n}}<\frac{\pi}{2}<\tau_{\bar{n}+1}
$$

(b) For $k=1,2, \ldots, \bar{n}$,

$$
\tau_{k}=\frac{j_{1, k}}{N}\left(1+\varepsilon_{1}\right),
$$

where

$$
\left|\varepsilon_{1}\right| \leq \frac{1}{2 \pi}\left(1-\frac{3}{\pi}\right) N^{-2}=0.0072 N^{-2} .
$$

Proof. (a) From (2.9) and the well-known inequality [17, p. 492]

$$
\left(k+\frac{1}{8}\right) \pi<j_{1, k}<\left(k+\frac{1}{4}\right) \pi,
$$

we have

$$
\tau_{n}<\frac{j_{1, n}}{N}<\frac{1}{N}\left(\bar{n}+\frac{1}{4}\right) \pi<\frac{N-1 / 2}{N} \cdot \frac{\pi}{2}<\frac{\pi}{2}
$$


and $\tau_{\bar{n}+1}<\frac{2}{3} \pi$ if $N \geq 5$. The first inequality in (2.22) and the monotonicity of $f_{1}(\theta)$ in the interval $(0, \pi)$ yield

$$
\begin{aligned}
\tau_{\bar{n}+1} & >\frac{j_{1, \bar{n}+1}}{N}-\frac{1}{16 N^{2}} f_{1}\left(\frac{2}{3} \pi\right) \\
& >\left(\bar{n}+\frac{9}{8}\right) \frac{\pi}{N}-\frac{1}{16 N^{2}}\left[\sqrt{3}-3\left(\frac{3}{\pi}-\frac{1}{\sqrt{3}}\right)\right]>\frac{\pi}{2}
\end{aligned}
$$

if $N \geq 5$.

(b) Equation (2.9) gives

$$
\tau_{k}=\frac{j_{1, k}}{N}-\frac{1}{16 N^{2}} f_{1}\left(\tau_{k}\right)
$$

Since $f_{1}(\theta)$ is positive and $(1 / \theta) f_{1}(\theta)$ is monotonically increasing in $(0, \pi / 2]$, it follows that

$$
0<\frac{1}{16 N^{2}} f_{1}\left(\tau_{k}\right)<\frac{1}{2 \pi}\left(1-\frac{3}{\pi}\right) \frac{\tau_{k}}{N^{2}}<\frac{1}{2 \pi}\left(1-\frac{3}{\pi}\right) \frac{j_{1, k}}{N^{3}}
$$

$k=1,2, \ldots, \bar{n}$, which of course implies $(2.20)-(2.21)$. This completes the proof of the lemma.

To derive the estimate (1.14), we also require a bound for the error in the two-term McMahon asymptotic expansion for the zeros of Bessel functions; see Hethcote [9, p. 38]. The particular result which we need is stated in the lemma below.

LEMMA 2. For $k \geq 4$,

$$
j_{1, k}=\left(k+\frac{1}{4}\right) \pi-\frac{3}{8 \pi(k+1 / 4)}+\varepsilon_{2},
$$

where

$$
\left|\varepsilon_{2}\right| \leq 0.4576 k^{-2} \text {. }
$$

From the numerical values of $j_{1, k}, k=1,2,3$, it is easily verified that (2.25)-(2.26) in fact holds for all $k \geq 1$. With Lemma 2 , we can now prove the following results.

LEMma 3. The number $M_{k}=(-1)^{k} J_{0}\left(j_{1, k}\right)$ given in (1.8) satisfies

$$
M_{k}=\sqrt{\frac{2}{\pi}}\left(j_{1, k}^{-1 / 2}-\frac{3}{16} j_{1, k}^{-5 / 2}\right)+\varepsilon_{3},
$$

where

$$
\left|\varepsilon_{3}\right| \leq \begin{cases}0.0582 k^{-7 / 2} & (k \geq 2) \\ 0.0360 k^{-7 / 2} & (k \geq 25)\end{cases}
$$


Proof. First, we put $x=j_{1, k}$ in the asymptotic expansion [16, p. 193]

(2.29) $J_{0}(x)=\sqrt{\frac{2}{\pi x}}\left[\cos \left(x-\frac{\pi}{4}\right)+\frac{1}{8 x} \sin \left(x-\frac{\pi}{4}\right)\right.$

$$
\left.-\frac{1^{2} \cdot 3^{2}}{2 !(8 x)^{2}} \cos \left(x-\frac{\pi}{4}\right)\right]+\delta_{1}
$$

where

$$
\left|\delta_{1}\right| \leq \frac{1^{2} \cdot 3^{2} \cdot 5^{2}}{3 ! 8^{3}} \sqrt{\frac{2}{\pi}} x^{-7 / 2}=0.0732 \sqrt{\frac{2}{\pi}} x^{-7 / 2} .
$$

Next, we replace $j_{1, k}$ in the trigonometric functions in (2.29) by its asymptotic approximation (2.25). The result is

$$
\begin{aligned}
& M_{k}=\sqrt{\frac{2}{\pi}} j_{1, k}^{-1 / 2}\left\{\cos \left[\frac{3}{8 \pi\left(k+\frac{1}{4}\right)}-\varepsilon_{2}\right]\left(1-\frac{3^{2}}{2 ! 8^{2} j_{1, k}^{2}}\right)\right. \\
&\left.-\sin \left[\frac{3}{8 \pi\left(k+\frac{1}{4}\right)}-\varepsilon_{2}\right] \frac{1}{8 j_{1, k}}\right\}+(-1)^{k} \delta_{1} .
\end{aligned}
$$

Now we approximate the cosine and sine function, respectively, by their two-term and one-term Maclaurin expansion. This results in

(2.31) $M_{k}=\sqrt{\frac{2}{\pi}} j_{1, k}^{-1 / 2}\left[1-\frac{9}{128 \pi^{2}\left(k+\frac{1}{4}\right)^{2}}-\frac{9}{128 j_{1, k}^{2}}\right.$

$$
\left.-\frac{3}{64 \pi\left(k+\frac{1}{4}\right) j_{1, k}}\right]+\delta_{2} \text {, }
$$

where

$$
\left|\delta_{2}\right| \leq \begin{cases}\sqrt{\frac{2}{\pi}} 0.0723 k^{-7 / 2} & (k \geq 2) \\ \sqrt{\frac{2}{\pi}} 0.0448 k^{-7 / 2} & (k \geq 25)\end{cases}
$$

Finally, we rewrite (2.25) as

$$
\left(k+\frac{1}{4}\right) \pi=j_{1, k}+\left(\frac{3}{8 \pi\left(k+\frac{1}{4}\right)}-\varepsilon_{2}\right)
$$

to obtain

$$
\frac{1}{\left(k+\frac{1}{4}\right) \pi}=j_{1, k}^{-1}\left(1+\delta_{3}\right)
$$


with

$$
\left|\delta_{3}\right|<\left(\frac{3}{8 \pi\left(k+\frac{1}{4}\right)}-\varepsilon_{2}\right) j_{1, k}^{-1} .
$$

Since $j_{1, k}>\pi k$ by (2.22), we have from (2.26)

$$
\left|\delta_{3}\right|<\left(\frac{3}{8 \pi k}+\frac{0.4576}{k^{2}}\right) \frac{1}{\pi k} \leq \begin{cases}0.1108 k^{-2} & (k \geq 2), \\ 0.0438 k^{-2} & (k \geq 25) .\end{cases}
$$

The desired result (2.27) now follows upon substituting (2.32) into (2.31).

From (2.25), it is easily seen that

$$
j_{1, k}-j_{1, k-1}=\pi+\delta_{4},
$$

where

$$
\left|\delta_{4}\right| \leq \begin{cases}1.1061 k^{-2} & (k \geq 2) \\ 1.0382 k^{-2} & (k \geq 25) .\end{cases}
$$

Rewriting (2.33) as

$$
j_{1, k-1}=j_{1, k}\left(1-\frac{\pi+\delta_{4}}{j_{1, k}}\right)
$$

and using the binomial expansion, it can be shown that

$$
\int_{j_{1 k-1}}^{j_{1 k}} x^{-1 / 2} d x=\pi j_{1, k}^{-1 / 2}+\frac{\pi^{2}}{4} j_{1, k}^{-3 / 2}+\delta_{5}
$$

and

$$
\int_{j_{1 \wedge-1}}^{j_{1 k}} x^{-3 / 2} d x=\pi j_{1, k}^{-3 / 2}+\delta_{6}
$$

where

$$
\left|\delta_{5}\right| \leq \begin{cases}2.1972 k^{-5 / 2} & (k \geq 2) \\ 0.8258 k^{-5 / 2} & (k \geq 25)\end{cases}
$$

and

$$
\left|\delta_{6}\right| \leq \begin{cases}3.1103 k^{-5 / 2} & (k \geq 2), \\ 0.4743 k^{-5 / 2} & (k \geq 25) .\end{cases}
$$

A combination of (2.27), (2.35) and (2.36) gives the following result; cf. [6, Eq. (4.1)]. 
LEMMA 4. The number $M_{k}$ given in (1.8) satisfies

$$
M_{k}=\frac{\sqrt{2}}{\pi^{3 / 2}} \int_{j_{1, k-1}}^{j_{1, k}} x^{-1 / 2} d x-\frac{1}{2 \sqrt{2 \pi}} \int_{j_{1, k-1}}^{j_{1, k}} x^{-3 / 2} d x+\varepsilon_{4},
$$

where

$$
\left|\varepsilon_{4}\right| \leq \begin{cases}1.2161 k^{-5 / 2} & (k \geq 2) \\ 0.3144 k^{-5 / 2} & (k \geq 25)\end{cases}
$$

In exactly the same manner, one can prove the following analogues of Lemmas 1 to 4 above.

LemMA $1^{\prime}$. Let $\hat{f}(\theta)$ be the function defined by (2.15) and let $\hat{\tau}_{k}$ denote the root of the equation $\hat{f}(\theta)=j_{0, k}$, where $j_{0, k}$ is the $k$ th positive zero of $J_{0}(x)$. (a) If $\bar{m}=n-\bar{n}=n-[n / 2]$ then

$$
\hat{\tau}_{m}<\frac{\pi}{2}<\hat{\tau}_{m+1} \text {. }
$$

(b) For $k=1,2, \ldots, \bar{m}$,

$$
\hat{\tau}_{k}=\frac{j_{0, k}}{N} \quad\left(1+\hat{\varepsilon}_{1}\right)
$$

where $N=n+1$ and

$$
\left|\hat{\varepsilon}_{1}\right|<0.1085 N^{-2} \text { for } N \geq 50 .
$$

Lemma $2^{\prime}$. For $k \geq 4$,

$$
j_{0, k}=\left(k-\frac{1}{4}\right) \pi+\frac{1}{8 \pi\left(k-\frac{1}{4}\right)}+\hat{\varepsilon}_{2},
$$

where

$$
\left|\hat{\varepsilon}_{2}\right| \leq 0.3268 k^{-2} \text {. }
$$

LEMmA $3^{\prime}$. The number $\hat{M}_{k}=(-1)^{k+1} j_{0, k} J_{1}\left(j_{0, k}\right)$ given in (1.12) satisfies

$$
\hat{M}_{k}=\sqrt{\frac{2}{\pi}}\left(j_{0, k}^{1 / 2}+\frac{1}{16} j_{0, k}^{-3 / 2}\right)+\hat{\varepsilon}_{3}
$$

where

$$
\left|\hat{\varepsilon}_{3}\right| \leq \begin{cases}0.1251 k^{-5 / 2} & (k \geq 2) \\ 0.0819 k^{-5 / 2} & (k \geq 25)\end{cases}
$$


LEMMA $4^{\prime}$. The number $\hat{M}_{k}$ given in (1.12) satisfies

$$
\hat{M}_{k}=\frac{\sqrt{2}}{\pi^{3 / 2}} \int_{j_{0 k-1}}^{j_{0 k}} x^{1 / 2} d x+\frac{1}{2 \sqrt{2 \pi}} \int_{j_{0, k-1}}^{j_{0, k}} x^{-1 / 2} d x+\hat{\varepsilon}_{4},
$$

where

$$
\left|\hat{\varepsilon}_{4}\right| \leq \begin{cases}1.3468 k^{-3 / 2} & (k \geq 2) \\ 0.4916 k^{-3 / 2} & (k \geq 25)\end{cases}
$$

An immediate consequence of $(2.48)$ is that the constant $D_{0}$ in (1.11) is in absolute value less than 2.6945 , a result which is needed later in our discussion. To see this, we recall that in [6, Eqs. (1.16) and (6.17)], it was shown that $D_{0}$ has the alternative expression

$$
D_{0}=\sum_{k=1}^{\infty}\left[\hat{M}_{k}-\frac{\sqrt{2}}{\pi^{3 / 2}} \int_{j_{0 k-1}}^{j_{0 k}} x^{1 / 2} d x-\frac{1}{2 \sqrt{2 \pi}} \int_{j_{0, k-1}}^{j_{0, k}} x^{-1 / 2} d x\right]
$$

The first term of the series can be calculated numerically. Thus, from (2.48), it follows that

$$
\left|D_{0}\right| \leq 0.0009+1.3468 \sum_{k=2}^{\infty} k^{-3 / 2}<2.6945 .
$$

3. Sketch of the procedure. Returning to (1.4), we have

$$
L_{n}=(n+1) \int_{0}^{\pi} \sin \frac{\theta}{2} \cos \frac{\theta}{2}\left|P_{n}^{(1,0)}(\cos \theta)\right| d \theta
$$

Throughout the remainder of this paper, we shall let $N=n+1$ and suppose that $N \geq 50$. In view of the identity (2.14), $L_{n}$ can be written as

$$
L_{n}=N\left[L_{n}^{(1)}+L_{n}^{(2)}\right]
$$

with

$$
\begin{aligned}
L_{n}^{(1)} & =\int_{0}^{\pi / 2} \sin \frac{\theta}{2} \cos \frac{\theta}{2}\left|P_{n}^{(1,0)}(\cos \theta)\right| d \theta, \\
L_{n}^{(2)} & =\int_{0}^{\pi / 2} \sin \frac{\theta}{2} \cos \frac{\theta}{2}\left|P_{n}^{(0,1)}(\cos \theta)\right| d \theta .
\end{aligned}
$$

We shall first be concerned with the constant $L_{n}^{(1)}$. The calculation of $L_{N}^{(2)}$ proceeds in a similar manner.

In (3.3), we replace the Jacobi polynomial $P_{n}^{(1,0)}(\cos \theta)$ by its uniform approximation given in (2.10). Note that the function

$$
g(\theta)=\left(\frac{\theta}{2} \cot \frac{\theta}{2}\right)^{1 / 2}
$$


is positive and bounded by 1 on the interval $0<\theta<\pi$, and that the function $f_{1}(\theta)$ in $(2.9)$ satisfies $(1 / \theta) f_{1}(\theta)<f_{1}^{\prime}(\theta)$ for $0<\theta<\pi$. Thus

$$
\left(\frac{f(\theta)}{f^{\prime}(\theta)}\right)^{1 / 2}\left(\cot \frac{\theta}{2}\right)^{1 / 2} \leq \sqrt{2}
$$

By Corollary 1, we have

$$
L_{n}^{(1)}=\frac{1}{\sqrt{2}} \int_{0}^{\pi / 2}\left(\frac{f(\theta)}{f^{\prime}(\theta)}\right)^{1 / 2}\left(\cot \frac{\theta}{2}\right)^{1 / 2}\left|J_{1}(f(\theta))\right| d \theta+\varepsilon_{5},
$$

where

$$
\left|\varepsilon_{5}\right| \leq 0.0059 N^{-5 / 2} \text {. }
$$

Let $\tau_{k}$ have the same meaning as given in Lemma 1 , and for convenience, put $\tau_{0} \equiv 0$. Since $\left|J_{1}(x)\right|=(-1)^{k} J_{1}(x)$ for $x$ in $\left(j_{1, k}, j_{1, k+1}\right)$ and $k=0,1,2, \ldots,(3.7)$ can be expressed as

$$
L_{n}^{(1)}=\frac{1}{\sqrt{2}}\left[I_{0}+\sum_{k=1}^{n-1}(-1)^{k} I_{k}+(-1)^{n} I_{\bar{n}}\right]+\varepsilon_{5},
$$

where

$$
\begin{aligned}
& \text { (3.10) } I_{k}=\int_{\tau_{k}}^{\tau_{k+1}}\left(\frac{f(\theta)}{f^{\prime}(\theta)}\right)^{1 / 2}\left(\cot \frac{\theta}{2}\right)^{1 / 2} J_{1}(f(\theta)) d \theta \text {, } \\
& k=0,1, \ldots, \bar{n}-1 \text {, }
\end{aligned}
$$

and

$$
I_{n}=\int_{\tau_{n}}^{\pi / 2}\left(\frac{f(\theta)}{f^{\prime}(\theta)}\right)^{1 / 2}\left(\cot \frac{\theta}{2}\right)^{1 / 2} J_{1}(f(\theta)) d \theta, \quad \bar{n}=\left[\frac{n}{2}\right]
$$

In the next section, it will be shown that

$$
\sum_{k=1}^{n-1}(-1)^{k} I_{k}=\frac{2^{3 / 2}}{N} S_{n}^{*}+\frac{\sqrt{2}}{N}\left[g\left(\tau_{1}\right) M_{1}-g\left(\tau_{\bar{n}}\right) M_{\tilde{n}}\right]+\varepsilon_{6},
$$

where

$$
S_{n}^{*}=\sum_{k=2}^{\bar{n}} g\left(\tau_{k}\right) M_{k}
$$

and

$$
\left|\varepsilon_{6}\right| \leq \sqrt{2} \times 0.9015 N^{-5 / 2}
$$


$M_{k}$ being the constant given in (1.8); cf. [6, p. 399]. The calculations of $I_{0}$ and $I_{\bar{n}}$ are also given in $\S 4$, and it will be proved that

$$
I_{0}=\frac{\sqrt{2}}{N} \int_{0}^{j_{1.1}} J_{1}(y) d y+\varepsilon_{7}
$$

and

$$
(-1)^{\bar{n}} I_{\bar{n}}=\frac{\sqrt{2}}{N}\left[g\left(\tau_{\bar{n}}\right) M_{\bar{n}}-(-1)^{n} g\left(\frac{\pi}{2}\right) J_{0}\left(f\left(\frac{\pi}{2}\right)\right)\right]+\varepsilon_{8}
$$

Here

$$
\left|\varepsilon_{7}\right| \leq \sqrt{2} \times 0.0442 N^{-5 / 2}
$$

and

$$
\left|\varepsilon_{8}\right| \leq \sqrt{2} \times 0.1088 N^{-5 / 2} .
$$

The following lemma is demonstrated in $\S 5$.

LEMMA 5. The sum $S_{n}^{*}$ in (3.13) has the asymptotic approximation

$$
\begin{aligned}
S_{n}^{*}= & B_{0}^{(1)}-\frac{\sqrt{2}}{\pi^{3 / 2}} \int_{0}^{j_{11}} x^{-1 / 2} d x \\
& +\frac{\sqrt{2}}{\pi^{3 / 2}} N^{1 / 2}\left(\int_{0}^{\pi}-\int_{j_{1, n} / N}^{\pi / 2}\right) g(\theta) \theta^{-1 / 2} d \theta \\
& +\frac{1}{2 \sqrt{\pi}} N^{-1 / 2}+\varepsilon_{9},
\end{aligned}
$$

where $B_{0}^{(1)}$ is a constant given by

$$
B_{0}^{(1)}=\sum_{k=2}^{\infty}\left(M_{k}-\frac{\sqrt{2}}{\pi^{3 / 2}} \int_{j_{1 k-1}}^{j_{1, k}} x^{-1 / 2} d x\right)
$$

and

$$
\left|\varepsilon_{9}\right| \leq 3.7924 n^{-3 / 2}
$$

The asymptotic approximation of $L_{n}^{(1)}$ is obtained by inserting (3.19) in (3.12), and combining the resulting expression with (3.15) and (3.16). First, we observe that the function $g(\theta)$ in (3.5) satisfies $g(0)=1$ and $g^{\prime}(0)=0$. Thus,

$$
g\left(\tau_{1}\right)=1+\frac{g^{\prime \prime}(\xi)}{2 !} \tau_{1}^{2}
$$


where $0<\xi<\tau_{1}<j_{1,1} / N<5 \pi / 4 N$. Furthermore, since $g^{\prime \prime}(\theta)$ is negative and decreasing in $(0, \pi)$, we have

$$
g\left(\tau_{1}\right) M_{1}=M_{1}+\varepsilon_{10}
$$

with

$$
\left|\varepsilon_{10}\right| \leq \frac{1}{2}\left|g^{\prime \prime}\left(\frac{5 \pi}{4 N}\right)\right|\left(\frac{j_{1,1}}{N}\right)^{2} M_{1} \leq 0.2465 N^{-2} .
$$

Substituting (3.19) and (3.23) in (3.12), we obtain

$$
\begin{aligned}
\sum_{k=1}^{n-1}(-1)^{k} I_{k}=\frac{\sqrt{2}}{N} & {\left[\left(2 B_{0}^{(1)}-\frac{2^{3 / 2}}{\pi^{3 / 2}} \int_{0}^{j_{11}} x^{-1 / 2} d x+M_{1}\right)\right.} \\
& +\frac{2^{3 / 2}}{\pi^{3 / 2}} N^{1 / 2}\left(\int_{0}^{\pi / 2}-\int_{j_{1, n} / N}^{\pi / 2}\right) g(\theta) \theta^{-1 / 2} d \theta \\
& \left.+\frac{1}{\sqrt{\pi}} N^{-1 / 2}+\varepsilon_{11}\right] \\
& -\frac{\sqrt{2}}{N} g\left(\tau_{\bar{n}}\right) M_{\bar{n}},
\end{aligned}
$$

where

$$
\left|\varepsilon_{11}\right| \leq 8.5212 n^{-3 / 2} \text {. }
$$

Next, we observe that $B_{0}^{(1)}$ is related to $B_{0}$ via the identity

$$
B_{0}=2 B_{0}^{(1)}-\frac{2^{3 / 2}}{\pi^{3 / 2}} \int_{0}^{j_{1,1}} x^{-1 / 2} d x+M_{1}+\int_{0}^{j_{1,1}} J_{1}(y) d y
$$

see Eqs. (1.10), (6.15) and (6.4) in [6]. A combination of (3.9), (3.15), (3.16) and (3.25) gives

$$
\text { (3.28) } \begin{aligned}
N L_{n}^{(1)}= & B_{0}+\frac{2^{3 / 2}}{\pi^{3 / 2}} N^{1 / 2}\left(\int_{0}^{\pi / 2}-\int_{j_{1 . n} / N}^{\pi / 2}\right) g(\theta) \theta^{-1 / 2} d \theta \\
& +\frac{1}{\sqrt{\pi}} N^{-1 / 2}-(-1)^{\bar{n}} g\left(\frac{\pi}{2}\right) J_{0}\left(f\left(\frac{\pi}{2}\right)\right)+E_{1},
\end{aligned}
$$

where

$$
\left|E_{1}\right| \leq 8.6801 n^{-3 / 2}
$$


As mentioned earlier, the evaluation of $L_{n}^{(2)}$ proceeds in a similar fashion, and the result is

$$
\text { (3.30) } \begin{aligned}
N L_{n}^{(2)}= & \frac{D_{0}}{N}+\frac{\sqrt{2}}{\pi^{3 / 2}} N^{1 / 2}\left(\int_{0}^{\pi / 2}-\int_{j_{0 m} / N}^{\pi / 2}\right) \hat{g}(\theta) \theta^{1 / 2} d \theta \\
& +\frac{1}{\sqrt{\pi}} N^{-1 / 2}+(-1)^{\bar{m}} \frac{1}{2 N} \hat{g}\left(\frac{\pi}{2}\right) \hat{f}\left(\frac{\pi}{2}\right) J_{1}\left(\hat{f}\left(\frac{\pi}{2}\right)\right) \\
& +E_{2}
\end{aligned}
$$

where

$$
\hat{g}(\theta)=\left(\frac{2}{\theta} \tan \frac{\theta}{2}\right)^{1 / 2}
$$

and

$$
\left|E_{2}\right| \leq 4.4788 n^{-3 / 2} \text {. }
$$

Note that the bound for $E_{2}$ is only half of that for $E_{1}$. An explanation of this phenomenon and a brief summary of the calculation for $L_{n}^{(2)}$ can be found in $\S 6$. Asymptotic expansion (1.10) and its error bound (1.14) is obtained by adding the results for $L_{n}^{(1)}$ and $L_{n}^{(2)}$ together. This is done in $\S 7$.

We close this section with a proof of Szegö's conjecture. Let $(n+1)^{1 / 2}=n^{1 / 2}+\frac{1}{2} n^{-1 / 2}+\rho_{1},(n+1)^{-1 / 2}=n^{-1 / 2}+\rho_{2}$ and $(n+1)^{-1}=n^{-1}+\rho_{3}$. From (1.10), we have

$$
L_{n+1}-L_{n}=\sqrt{\frac{2}{\pi}} n^{-1 / 2}+\frac{2 \sqrt{2}}{\sqrt{\pi}} \rho_{1}+\sqrt{\frac{2}{\pi}} \rho_{2}+D_{0} \rho_{3}+\rho^{*},
$$

where $\rho^{*}=\varepsilon(n+1)-\varepsilon(n)$. It is easy to see that $\left|\rho_{1}\right| \leq \frac{1}{8} n^{-3 / 2}$, $\left|\rho_{2}\right| \leq \frac{1}{2} n^{-3 / 2}$ and $\left|\rho_{3}\right| \leq \frac{1}{7} n^{-3 / 2}$ for $n \geq 49$. By (1.14), we also have $\left|\rho^{*}\right| \leq 30 n^{-3 / 2}$ for $n \geq 49$. Thus

$$
L_{n+1}-L_{n}=\sqrt{\frac{2}{\pi}} n^{-1 / 2}+\rho
$$

for $n \geq 49$, where

$$
|\rho| \leq 30.9833 n^{-3 / 2} \text {. }
$$

Here use has been made of (2.51). The right-hand side of (3.33) is obviously positive if $n \geq 49$, thus proving (1.6) for all $n \geq 49$. 
TABLE OF $L_{n}(1 \leq n \leq 50)$

\begin{tabular}{c|c||c|c}
\hline & & & \\
$n$ & $L_{n}$ & $n$ & $L_{n}$ \\
\hline 1 & 1.6666666667 & 26 & 7.7071046904 \\
3 & 2.1757550766 & 27 & 7.8592773737 \\
4 & 2.6042945349 & 28 & 8.0087552924 \\
5 & 3.3225397887 & 30 & 8.3001685828 \\
& & & \\
6 & 3.6360053510 & 31 & 8.4423475116 \\
7 & 3.9277225676 & 32 & 8.5823211753 \\
8 & 4.2016761244 & 33 & 8.7201891188 \\
9 & 4.4607644000 & 34 & 8.8560436165 \\
10 & 4.7071738476 & 35 & 8.9899703935 \\
& & & \\
11 & 4.9426021800 & 36 & 9.1220492583 \\
12 & 5.1683989094 & 37 & 9.2523546614 \\
13 & 5.3856578313 & 38 & 9.3809561825 \\
14 & 5.5952801306 & 39 & 9.5079189674 \\
15 & 5.7980187723 & 40 & 9.6333041150 \\
& & & \\
16 & 5.9945105436 & 41 & 9.7571690148 \\
17 & 6.1852997023 & 42 & 9.8795676628 \\
18 & 6.3708557767 & 43 & 10.0005509311 \\
19 & 6.5515871874 & 44 & 10.1201668153 \\
20 & 6.7278518354 & 45 & 10.2384606609 \\
& & & \\
21 & 6.8999654408 & 46 & 10.3554753584 \\
22 & 7.0682081922 & 47 & 10.4712515292 \\
23 & 7.2328301049 & 48 & 10.5858276893 \\
24 & 7.3940553830 & 49 & 10.6992403971 \\
25 & 7.5520860056 & 50 & 10.8115243935 \\
\hline
\end{tabular}

4. Calculation of $I_{k}$. In view of the identity $J_{1}(x)=-J_{0}^{\prime}(x)$, the integral $I_{k}$ in (3.10) can be expressed as

$$
I_{k}=\int_{\tau_{k}}^{\tau_{k+1}} G(\theta) d\left(-J_{0}(f(\theta))\right)
$$

where

$$
G(\theta)=\left(\frac{2 f(\theta)}{\theta}\right)^{1 / 2}\left[f^{\prime}(\theta]^{-3 / 2} g(\theta)\right.
$$


and $g(\theta)$ has the same meaning as given in (3.5). Using the monotonicity property of $(1 / \theta) f_{1}(\theta)$ and $f_{1}^{\prime}(\theta)$ on $(0, \pi / 2)$, it is easily seen that

$$
\left(\frac{f(\theta)}{\theta}\right)^{1 / 2}=N^{1 / 2}\left(1+\delta_{7}\right)
$$

and

$$
\left[f^{\prime}(\theta)\right]^{-3 / 2}=N^{-3 / 2}\left(1+\delta_{8}\right),
$$

where $\left|\delta_{7}\right| \leq 0.0036 N^{-2}$ and $\left|\delta_{8}\right| \leq 0.0405 N^{-2}$. Thus,

$$
G(\theta)=\sqrt{2} g(\theta) N^{-1}+\varepsilon_{12}
$$

with

$$
\left|\varepsilon_{12}\right| \leq \sqrt{2} \times 0.0442 N^{-3} \text { for } N \geq 50 .
$$

Inserting (4.5) in (4.1) and applying an integration by parts, we obtain

$$
\begin{aligned}
I_{k}= & (-1)^{k} \frac{\sqrt{2}}{N}\left[g\left(\tau_{k}\right) M_{k}+g\left(\tau_{k+1}\right) M_{k+1}\right] \\
& +\frac{\sqrt{2}}{N} \int_{\tau_{k}}^{\tau_{k+1}} g^{\prime}(\theta) J_{0}(f(\theta)) d \theta+\int_{\tau_{k}}^{\tau_{k+1}} \varepsilon_{12} J_{1}(f(\theta)) d f(\theta),
\end{aligned}
$$

where $M_{k}$ is given in (1.8). By (4.6), the last integral on the right is in absolute value less than $\sqrt{2} \times 0.0442 N^{-3}\left(M_{k}+M_{k+1}\right)$. Thus, upon summation, we have

$$
\begin{aligned}
\sum_{k=1}^{n-1}(-1)^{k} I_{k}= & \frac{2 \sqrt{2}}{N} S_{n}^{*}+\frac{\sqrt{2}}{N}\left[g\left(\tau_{1}\right) M_{1}-g\left(\tau_{\tilde{n}}\right) M_{\tilde{n}}\right] \\
& +\frac{\sqrt{2}}{N} \sum_{k=1}^{\bar{n}-1}(-1)^{k} \int_{\tau_{k}}^{\tau_{k+1}} g^{\prime}(\theta) J_{0}(f(\theta)) d \theta+\varepsilon_{13},
\end{aligned}
$$

where $S_{n}^{*}$ is given in (3.13) and

$$
\left|\varepsilon_{13}\right| \leq \sqrt{2} \times 0.0442 N^{-3}\left[2 \sum_{k=2}^{\bar{n}-1} M_{k}+M_{1}+M_{\tilde{n}}\right] ;
$$

cf. Eq. (3.12). Since $j_{1, k} \geq \pi k$, Lemma 3 gives

$$
M_{k} \leq \sqrt{\frac{2}{\pi}}\left(\frac{1}{\sqrt{\pi}} k^{-1 / 2}+\frac{3}{16 \pi^{5 / 2}} k^{-5 / 2}\right)+\left|\varepsilon_{3}\right| .
$$

Furthermore, since

$$
\sum_{k=2}^{n-1} k^{-p} \leq \int_{1}^{\bar{n}-1} x^{-p} d x, \quad p>1,
$$


by (2.28) we have

$$
\begin{aligned}
\left|\varepsilon_{13}\right| \leq \sqrt{2} \times 0.0442 N^{-3}\left[\frac{4 \sqrt{2}}{\pi}\right. & (\bar{n}-1)^{1 / 2}+\frac{\sqrt{2}}{4 \pi^{3}}+2 \times 0.0582 \times \frac{2}{5} \\
& \left.+\left|J_{0}\left(j_{1,1}\right)\right|+\frac{2}{\pi}\left(1-\frac{2}{N}\right)^{-1 / 2} N^{-1 / 2}\right] .
\end{aligned}
$$

Thus,

$$
\left|\varepsilon_{13}\right| \leq \sqrt{2} \times 0.0597 N^{-5 / 2} .
$$

Let us now estimate the second last term in (4.8), namely,

$$
\varepsilon_{14}=\frac{\sqrt{2}}{N} \sum_{k=1}^{n-1}(-1)^{k} \int_{\tau_{k}}^{\tau_{k+1}} g^{\prime}(\theta) J_{0}(f(\theta)) d \theta .
$$

In the integral, we make the change of variable $f(\theta)=y$. This gives

$$
\int_{\tau_{k}}^{\tau_{k+1}} g^{\prime}(\theta) J_{0}(f(\theta)) d \theta=\int_{j_{l, k}}^{j_{1, k+1}} H(y) y J_{0}(y) d y,
$$

where

$$
H(y)=\frac{g^{\prime}(\theta)}{f^{\prime}(\theta) f(\theta)} .
$$

By the Mean-Value Theorem,

$$
H(y)=H\left(j_{0, k+1}\right)+H^{\prime}\left(\xi_{k}\right)\left(y-j_{0, k+1}\right)
$$

for some $\xi_{k}$ between $y$ and $j_{0, k+1}$. Inserting (4.15) in (4.13), we obtain

$$
\int_{\tau_{k}}^{\tau_{k+1}} g^{\prime}(\theta) J_{0}(f(\theta)) d \theta=\int_{j_{1, k}}^{j_{1, k+1}} H^{\prime}\left(\xi_{k}\right)\left(y-j_{0, k+1}\right) y J_{0}(y) d y
$$

the first term vanishing in view of the identity $y J_{0}(y)=\left(y J_{1}(y)\right)^{\prime}$. To estimate $H^{\prime}(y)$, we note that both $g^{\prime}(\theta)$ and $g^{\prime \prime}(\theta)$ are negative and decreasing. Furthermore, $g^{\prime}(0)=0$ and $\left|(1 / \theta) g^{\prime}(\theta)\right|_{\theta=0} \leq\left|g^{\prime \prime}(\pi / 2)\right|$. Thus, for $\tau_{k} \leq \theta \leq \tau_{k+1}$, or equivalently for $j_{1, k} \leq y \leq j_{1, k+1}$,

$$
\left|H^{\prime}(y)\right| \leq 0.1503 N^{-2} j_{1, k}^{-1} \text {. }
$$

Using (2.22) and

$$
\left(k-\frac{1}{4}\right) \pi<j_{0, k}<\left(k-\frac{1}{8}\right) \pi,
$$

we also have $\left|y-j_{0, k+1}\right| \leq 3 \pi / 4$ and $0<j_{1, k+1}-j_{1, k} \leq 9 \pi / 8$. A combination of these results shows that the integral in (4.16) is bounded by

$$
0.1503 N^{-2} \cdot \frac{27}{32} \sqrt{2} \pi^{3 / 2}\left(j_{1, k}^{-1 / 2}+\frac{9}{32} \pi j_{1, k}^{-3 / 2}\right)
$$


Therefore the error term $\varepsilon_{14}$ given in (4.12) satisfies

$$
\left|\varepsilon_{14}\right|<\frac{\sqrt{2}}{N} \times 0.1793 \pi N^{-2}\left(\sum_{k=1}^{n-1} k^{-1 / 2}+\frac{9}{32} \sum_{k=1}^{n-1} k^{-3 / 2}\right) .
$$

This together with (4.10) yields

$$
\left|\varepsilon_{14}\right| \leq \sqrt{2} \times 0.8418 N^{-5 / 2} \text { for } N \geq 50
$$

Coupling (4.11) and (4.20), we obtain (3.14), thus proving the asymptotic approximation (3.12).

The calculation of $I_{0}$ proceeds in a slightly different manner. We first make the change of variable $y=f(\theta)$. Thus

$$
I_{0}=\int_{0}^{j_{1.1}} G(\theta) J_{1}(y) d y .
$$

Substituting (4.5) in (4.21) yields

$$
I_{0}=\frac{\sqrt{2}}{N} \int_{0}^{j_{11}} g(\theta) J_{1}(y) d y+\varepsilon_{15}
$$

where

$$
\left|\varepsilon_{15}\right| \leq \sqrt{2} \times 0.0088 N^{-5 / 2} .
$$

Note that $g(\theta)$ in (4.22) is a function of $y$ and

$$
\frac{d^{2} g}{d y^{2}}=\left(\frac{g^{\prime}(\theta)}{f^{\prime}(\theta)}\right)^{\prime} \frac{1}{f^{\prime}(\theta)}
$$

Using the facts that both $g^{\prime}$ and $g^{\prime \prime}$ are negative and decreasing in $(0, \pi / 2]$ and $g^{\prime}(0)=0$, we have

$$
\left|\frac{d^{2} g}{d y^{2}}\right| \leq N^{-2}\left|g^{\prime \prime}\left(\frac{5 \pi}{4 N}\right)\right| \leq 0.0835 N^{-2}
$$

for $0 \leq y \leq j_{1,1}$ and $N \geq 50$. Expanding $g(\theta)$ at $y=0$ gives

$$
g(\theta)=1+\frac{1}{2}\left(\left.\frac{d^{2} g}{d y^{2}}\right|_{y=\eta}\right) y^{2}
$$

for some $\eta$ between 0 and $y$. Inserting (4.25) in (4.22) and making use of (4.24), we obtain the required result (3.15).

The calculation of $I_{n}$ resembles that of $I_{k}$ for $1 \leq k \leq \bar{n}-1$. Thus, instead of (4.7), we have

$$
(-1)^{n} I_{\bar{n}}=\frac{\sqrt{2}}{N}\left[g\left(\tau_{\bar{n}}\right) M_{\bar{n}}-(-1)^{n} g\left(\frac{\pi}{2}\right) J_{0}\left(f\left(\frac{\pi}{2}\right)\right)\right]+\varepsilon_{8}
$$


where

$$
\begin{aligned}
\varepsilon_{8}= & (-1)^{\bar{n}} \frac{\sqrt{2}}{N} \int_{\tau_{n}}^{\pi / 2} g^{\prime}(\theta) J_{0}(f(\theta)) d \theta \\
& +(-1)^{\bar{n}} \int_{\tau_{n}}^{\pi / 2} \varepsilon_{12} J_{1}(f(\theta)) d f(\theta)
\end{aligned}
$$

cf. (3.16). By (4.6), the second integral on the right is less than $\sqrt{2} \times 0.0442 N^{-3}\left|J_{0}(f(\pi / 2))-J_{0}\left(j_{1, n}\right)\right|$. Using (2.22) and the facts that $\left|J_{0}(x)\right| \leq \sqrt{2 / \pi x}$ and $f(\theta) \geq N \theta$, it can easily be shown that

$$
\left|(-1)^{n} \int_{\tau_{n}}^{\pi / 2} \varepsilon_{12} J_{1}(f(\theta)) d f(\theta)\right| \leq \sqrt{2} \times 0.0011 N^{-5 / 2}
$$

The first integral in (4.27) can be estimated as in (4.13)-(4.19). Instead of (4.15), here we expand $H(y)$ at $y=f(\pi / 2)$. The analogue of (4.16) is

$$
\begin{aligned}
& (-1)^{n} \int_{\tau_{n}}^{\pi / 2} g^{\prime}(\theta) J_{0}(f(\theta)) d \theta \\
& =(-1)^{\bar{n}} H\left(f\left(\frac{\pi}{2}\right)\right) f\left(\frac{\pi}{2}\right) J_{1}\left(f\left(\frac{\pi}{2}\right)\right) \\
& \quad+(-1)^{n} \int_{j_{1 n}}^{f(\pi / 2)} H^{\prime}\left(\xi_{\bar{n}}\right)\left(y-f\left(\frac{\pi}{2}\right)\right) y J_{0}(y) d y
\end{aligned}
$$

for some $\xi_{n}$ between $y$ and $f(\pi / 2)$. Again using the facts that $\left|J_{1}(x)\right| \leq$ $\sqrt{2 / \pi x}$ and $f(\pi / 2) \geq(\pi / 2) N$, it can be shown that the first term on the right is bounded by $0.1025 N^{-3 / 2}$. By an argument similar to that for (4.17), we also have

$$
\left|H^{\prime}\left(\xi_{n}\right)\right| \leq 0.0350 N^{-3}
$$

This, together with

$$
\left|y-f\left(\frac{\pi}{2}\right)\right| \leq f\left(\frac{\pi}{2}\right)-j_{1, \bar{n}}<\frac{1}{4 N}\left(1-\frac{3}{\pi}\right)+\frac{7}{8} \pi
$$

implies that the second term on the right-hand side of (4.29) is bounded by $0.0052 N^{-3 / 2}$. Therefore,

$$
\left|\frac{\sqrt{2}}{N} \int_{\tau_{\hbar}}^{\pi / 2} g^{\prime}(\theta) J_{0}(f(\theta)) d \theta\right| \leq \sqrt{2} \times 0.1077 N^{-5 / 2} .
$$

The estimate (3.18) now follows from (4.28) and (4.30). This completes the proof of (3.16). 
5. Proof of Lemma 5. From (3.13), we have

$$
S_{n}^{*}=\sum_{k=2}^{n} M_{k}+\sum_{k=2}^{n}\left[g\left(\tau_{k}\right)-1\right] M_{k}
$$

Replacing $M_{k}$ by its asymptotic approximation in Lemma 4, we can express $S_{n}^{*}$ as

$$
S_{n}^{*}=S_{n, 1}+S_{n, 2}^{*}+S_{n, 3}^{*}+\varepsilon_{16}
$$

where

$$
S_{n, 1}=\sum_{k=2}^{\infty}\left(M_{k}-\frac{\sqrt{2}}{\pi^{3 / 2}} \int_{j_{1, k-1}}^{j_{1, k}} x^{-1 / 2} d x+\frac{1}{2 \sqrt{2 \pi}} \int_{j_{1, k-1}}^{j_{1, k}} x^{-3 / 2} d x\right)
$$

$$
\begin{gathered}
S_{n, 2}^{*}=\frac{\sqrt{2}}{\pi^{3 / 2}} \sum_{k=2}^{n} \int_{j_{1, k-1}}^{j_{1, k}} g\left(\tau_{k}\right) x^{-1 / 2} d x, \\
S_{n, 3}^{*}=-\frac{1}{2 \sqrt{2 \pi}} \sum_{k=2}^{n} \int_{j_{1, k-1}}^{j_{1 k}} g\left(\tau_{k}\right) x^{-3 / 2} d x,
\end{gathered}
$$

and

$$
\varepsilon_{16}=\sum_{k=2}^{n}\left[g\left(\tau_{k}\right)-1\right] \varepsilon_{4}+\sum_{k=n+1}^{\infty} \varepsilon_{4}
$$

Since $g^{\prime \prime}(\theta)$ is negative and decreasing in $[0, \pi / 2]$, it follows from (3.22) and (2.23) that

$$
\left.\mid g\left(\tau_{k}\right)-1\right)\left|\leq \frac{1}{2}\right| g^{\prime \prime}\left(\frac{\pi}{2}\right) \mid\left(j_{1, k} / N\right)^{2}
$$

A combination of (2.40), (2.22) and (4.10) gives

$$
\left|\varepsilon_{16}\right| \leq 2.2259 n^{-3 / 2} \text { for } n \geq 49 \text {. }
$$

Note that the infinite series $S_{n, 1}$ is absolutely convergent by Lemma 4, and is a constant independent of $n$; cf. [6, p. 405].

(A) Evaluation of $S_{n, 2}^{*}$.

The argument here parallels that given for $S_{n, 2}$ in [6, p. 405], except that the zeros $\theta_{k}$ of the Jacobi polynomial there is replaced by the roots $\tau_{k}$ of the equation $f(\theta)=j_{1, k}$ and the $O$-terms are replaced by explicit bounds. Thus, in (5.3), we make the change of variable $x=N \theta$ and write

$$
g\left(\tau_{k}\right)=g(\theta)+g^{\prime}(\theta)\left(\tau_{k}-\theta\right)+\frac{1}{2} g^{\prime \prime}(\xi)\left(\tau_{k}-\theta\right)^{2},
$$


where $\xi$ is between $\tau_{k}$ and $\theta$. Since

$$
\left|g^{\prime \prime}(\xi)\right| \leq\left|g^{\prime \prime}(\pi / 2)\right| \text { and }\left(\tau_{k}-\theta\right)^{2} \leq\left(j_{1, k}-j_{1, k-1}\right)^{2} / N^{2}
$$

for $\theta \in\left[j_{1, k-1} / N, j_{1, k} / N\right]$, the remainder term $\frac{1}{2} g^{\prime \prime}(\xi)\left(\tau_{k}-\theta\right)^{2}$ in (5.7) contributes to $S_{n, 2}^{*}$ an error

$$
\left|\varepsilon_{17}\right| \leq 0.3171 \sqrt{\pi} n^{1 / 2} N^{-2} \text { for } N \geq 50 .
$$

Here use has been made of (2.33)-(2.34). Substituting (5.7) in (5.3) then gives

$$
S_{n, 2}^{*}=\frac{\sqrt{2}}{\pi^{3 / 2}}\left[S_{n, 2}^{(1)^{*}}+S_{n, 2}^{(2)^{*}}\right]+\varepsilon_{17}
$$

where

$$
S_{n, 2}^{(1)^{*}}=N^{1 / 2} \sum_{k=2}^{\bar{n}} \int_{j_{1 k-1} / N}^{j_{1, k} / N} g(\theta) \theta^{-1 / 2} d \theta
$$

and

$$
S_{n, 2}^{(2)^{*}}=N^{1 / 2} \sum_{k=2}^{\bar{n}} \int_{j_{1,-1} / N}^{j_{1, k} / N}\left(\tau_{k}-\theta\right) g^{\prime}(\theta) \theta^{-1 / 2} d \theta .
$$

Clearly, $S_{n, 2}^{(1)^{*}}$ can be written as

$$
S_{n, 2}^{(1)^{*}}=N^{1 / 2}\left(\int_{0}^{\pi / 2}-\int_{0}^{j_{1,1} / N}-\int_{j_{1, n} / N}^{\pi / 2}\right) g(\theta) \theta^{-1 / 2} d \theta .
$$

For $S_{n, 2}^{(2)^{*}}$, we let $\Phi(\theta)=g^{\prime}(\theta) \theta^{-1 / 2}$ and write

$$
\tau_{k}-\theta=\left[\tau_{k}-\frac{1}{2 N}\left(j_{1, k}+j_{1, k-1}\right)\right]-\left[\theta-\frac{1}{2 N}\left(j_{1, k}+j_{1, k-1}\right)\right] \text {. }
$$

Then

$$
\begin{aligned}
S_{n, 2}^{(2)^{*}}= & N^{1 / 2} \sum_{k=2}^{n}\left(\tau_{k}-\frac{j_{1, k}+j_{1, k-1}}{2 N}\right) \int_{j_{1 k-1} / N}^{j_{1 k} / N} \Phi(\theta) d \theta \\
& -N^{1 / 2} \sum_{k=2}^{\bar{n}} \int_{j_{1, k-1} / N}^{j_{1, k} / N}\left(\theta-\frac{j_{1, k}+j_{1, k-1}}{2 N}\right) \Phi(\theta) d \theta
\end{aligned}
$$

Making the change of variable $t=\theta-(1 / 2 N)\left(j_{1, k}+j_{1, k-1}\right)$, each integral in the second sum on the right becomes

$$
\begin{aligned}
\int_{0}^{\left(j_{1, k}-j_{1 k-1}\right) / 2 N} t\left[\Phi\left(t+\frac{j_{1, k}+j_{1, k-1}}{2 N}\right)\right. \\
\left.-\Phi\left(-t+\frac{j_{1, k}+j_{1, k-1}}{2 N}\right)\right] d t .
\end{aligned}
$$


By the mean value theorem, this integral is equal to

$$
\int_{0}^{\left(j_{1, k}-j_{1, k-1}\right) / 2 N} 2 t^{2} \Phi^{\prime}\left(\xi_{t}\right) d t
$$

for some $\xi_{t}$ satisfying

$$
\frac{1}{2 N}\left(j_{1, k}+j_{1, k-1}\right)-t<\xi_{t}<\frac{1}{2 N}\left(j_{1, k}+j_{1, k-1}\right)+t .
$$

Since $0 \leq t \leq\left(j_{1, k}-j_{1, k-1}\right) / 2 N$ in (5.15), we have $j_{1, k-1} / N \leq \xi_{t} \leq$ $j_{1, k} / N$. Furthermore, since $\left|\Phi^{\prime}\left(\xi_{t}\right)\right| \leq\left|g^{\prime \prime}(\pi / 2)\right| \xi_{t}^{-1 / 2}$, by using (2.33)(2.34), it can be shown that the integral in (5.15) is bounded by

$$
\frac{1}{12 \sqrt{\pi}} N^{-5 / 2}\left|g^{\prime \prime}\left(\frac{\pi}{2}\right)\right|\left(k-\frac{7}{8}\right)^{-1 / 2}\left(\pi+\frac{1.1061}{4}\right)^{3} \text {. }
$$

From this and (4.10), it follows that the second term on the right of (5.13), i.e.,

$$
\varepsilon_{18}=-N^{1 / 2} \sum_{k=2}^{n} \int_{j_{1, k-1} / N}^{j_{1, k} / N}\left(\theta-\frac{j_{1, k}+j_{1, k-1}}{2 N}\right) \Phi(\theta) d \theta
$$

satisfies

$$
\left|\varepsilon_{18}\right| \leq 0.0956 \pi^{3 / 2} N^{-2} n^{1 / 2} .
$$

To calculate the first term on the right-hand side of (5.13), we use Lemma 1 and (2.33). Thus

$$
\begin{gathered}
\sum_{k=2}^{\bar{n}}\left(\tau_{k}-\frac{j_{1, k}+j_{1, k-1}}{2 N}\right) \int_{j_{1, k-1} / N}^{j_{1, k} / N} g^{\prime}(\theta) \theta^{-1 / 2} d \theta \\
=\frac{\pi}{2 N} \sum_{k=2}^{\bar{n}} \int_{j_{1, k-1} / N}^{j_{1, k} / N} g^{\prime}(\theta) \theta^{-1 / 2} d \theta+\varepsilon_{19},
\end{gathered}
$$

where

$$
\varepsilon_{19}=\sum_{k=2}^{n}\left(\frac{\delta_{4}}{2 N}+\frac{j_{1, k}}{N} \varepsilon_{1}\right) \int_{j_{1, k-1} / N}^{j_{1 k} / N} g^{\prime}(\theta) \theta^{-1 / 2} d \theta .
$$

Since $(1 / \theta) g^{\prime}(\theta)$ is negative and decreasing in $[0, \pi / 2]$

$$
\frac{1}{\theta}\left|g^{\prime}(\theta)\right| \leq \frac{2}{\pi}\left|g^{\prime}\left(\frac{\pi}{2}\right)\right| \text {. }
$$

Using (2.22), (2.33) and (4.10), it can then be shown that

$$
\left|\varepsilon_{19}\right| \leq 0.0588 \sqrt{\pi} N^{-2} \text {. }
$$


Inserting (5.18) in (5.13) and adding the resulting expression to (5.12), we obtain from (5.9)

$$
\text { (5.21) } \begin{aligned}
S_{n, 2}^{*}= & \frac{\sqrt{2}}{\pi^{3 / 2}} N^{1 / 2}\left(\int_{0}^{\pi / 2}-\int_{0}^{j_{1,1} / N}-\int_{j_{1, n} / N}^{\pi / 2}\right) g(\theta) \theta^{-1 / 2} d \theta \\
& +\frac{1}{\sqrt{2 \pi}} N^{-1 / 2} \sum_{k=2}^{n} \int_{j_{1, k-1} / N}^{j_{1, k} / N} g^{\prime}(\theta) \theta^{-1 / 2} d \theta+\varepsilon_{20},
\end{aligned}
$$

where

$$
\varepsilon_{20}=\frac{\sqrt{2}}{\pi^{3 / 2}}\left[N^{1 / 2} \varepsilon_{19}+\varepsilon_{18}\right]+\varepsilon_{17}
$$

and by (5.8), (5.17) and (5.20)

$$
\left|\varepsilon_{20}\right| \leq 0.7237 n^{-3 / 2} \text { for } n \geq 49 \text {. }
$$

(B) Evaluation of $S_{n, 3}^{*}$.

The analysis here is similar to that of $S_{n, 2}^{*}$, and is in fact simpler. We first make the change of variable $x=N \theta$ in (5.4) and then replace $g\left(\tau_{k}\right)$ by $g(\theta)+g^{\prime}(\bar{\xi})\left(\tau_{k}-\theta\right)$; cf. (5.7). The result is

$$
s_{n, 3}^{*}=-\frac{1}{2 \sqrt{2 \pi}} N^{-1 / 2}\left[S_{n, 3}^{(1)^{*}}+\varepsilon_{21}\right]
$$

where

$$
S_{n, 3}^{(1)^{*}}=\sum_{k=2}^{\bar{n}} \int_{j_{1, k-1} / N}^{j_{1, k} / N} g(\theta) \theta^{-3 / 2} d \theta
$$

and

$$
\varepsilon_{21}=\sum_{k=2}^{n} \int_{j_{1, k-1} / N}^{j_{1, k} / N} g^{\prime}(\bar{\xi})\left(\tau_{k}-\theta\right) \theta^{-3 / 2} d \theta .
$$

$\bar{\xi}$ being between $\tau_{k}$ and $\theta$. By integration by parts

$$
\begin{aligned}
S_{n, 3}^{(1)^{*}=} & 2 g\left(\frac{j_{1,1}}{N}\right)\left(\frac{j_{1,1}}{N}\right)^{-1 / 2}-2 g\left(\frac{j_{1, \bar{n}}}{N}\right)\left(\frac{j_{1, \bar{n}}}{N}\right)^{-1 / 2} \\
& +2 \int_{j_{11} / N}^{j_{1 n} / N} g^{\prime}(\theta) \theta^{-1 / 2} d \theta .
\end{aligned}
$$

Note that the last integral on the right-hand side is equal to the finite sum in (5.21). Since $\left|g^{\prime \prime}(\theta)\right| \leq\left|g^{\prime \prime}(\pi / 2)\right|$ for $\theta \in[0, \pi / 2]$ and $j_{1,1}<$ $5 \pi / 4$, by a two-term Maclaurin expansion we have

$$
g\left(\frac{j_{1,1}}{N}\right)=1+\varepsilon_{22}, \quad\left|\varepsilon_{22}\right| \leq 1.1035 N^{-2} .
$$


Put $h(\theta)=(\cot (\theta / 2))^{1 / 2}$. Since $g(\theta) \theta^{-1 / 2}=(1 / \sqrt{2})(\cot (\theta / 2))^{1 / 2}$, by the mean-value theorem

$$
g\left(\frac{j_{1, \tilde{n}}}{N}\right)\left(\frac{j_{1, \tilde{n}}}{N}\right)^{-1 / 2}=\frac{1}{\sqrt{2}}\left[1+h^{\prime}(\eta)\left(\frac{j_{1, \bar{n}}}{N}-\frac{\pi}{2}\right)\right],
$$

where $\eta$ is between $j_{1, n} / N$ and $\pi / 2$. Furthermore, since $h^{\prime}(\theta)$ is negative and increasing in $(0,2 \pi / 3)$ and $\pi(1-7 / 4 N) / 2<j_{1, \bar{n}} / N$, we also have $\left|h^{\prime}(\eta)\right|<\left|h^{\prime}(\bar{\eta})\right|$, where $\bar{\eta}=\pi(1-7 / 4 N) / 2$. From this, we conclude that

$$
g\left(\frac{j_{1, \bar{n}}}{N}\right)\left(\frac{j_{1, \bar{n}}}{N}\right)^{-1 / 2}=\frac{1}{\sqrt{2}}\left(1+\varepsilon_{23}\right), \quad\left|\varepsilon_{23}\right| \leq 0.4504 \frac{\pi}{N}
$$

for $n \geq 50$. Inserting (5.27) and (5.29) in (5.26) and coupling the resulting expression with (5.23) gives

$$
\begin{aligned}
S_{n, 3}^{*}= & -\frac{1}{\sqrt{2 \pi}} j_{1,1}^{-1 / 2}+\frac{1}{2 \sqrt{\pi}} N^{-1 / 2} \\
& -\frac{1}{\sqrt{2 \pi}} N^{-1 / 2} \int_{j_{11} / N}^{j_{1 n} / N} g^{\prime}(\theta) \theta^{-1 / 2} d \theta+\varepsilon_{24},
\end{aligned}
$$

where

$$
\varepsilon_{24}=-\frac{1}{\sqrt{2 \pi}} j_{1,1}^{-1 / 2} \varepsilon_{22}+\frac{1}{2 \sqrt{\pi}} N^{-1 / 2} \varepsilon_{23}-\frac{1}{2 \sqrt{2 \pi}} N^{-1 / 2} \varepsilon_{21} .
$$

To estimate $\varepsilon_{21}$, we note that $j_{1, k-1} / N<\tau_{k}<j_{1, k} / N$ and

$$
\frac{1}{\theta}\left|g^{\prime}(\theta)\right|<\frac{2}{\pi}\left|g^{\prime}\left(\frac{\pi}{2}\right)\right| \quad \text { for } \theta \in\left[0, \frac{\pi}{2}\right] \text {. }
$$

Thus, as in (5.20), it can be shown that

$$
\left|\varepsilon_{21}\right| \leq \sqrt{\pi} \cdot 1.1162 N^{-3 / 2} n^{1 / 2}
$$

A combination of (5.27), (5.29) and (5.31) yields

$$
\left|\varepsilon_{24}\right| \leq 0.8256 n^{-3 / 2} \text { for } n \geq 49 \text {. }
$$

Observing that the sum $S_{n, 1}$ in (5.2) can be written as

$$
S_{n, 1}=B_{0}^{(1)}+\frac{1}{\sqrt{2 \pi}} j_{1,1}^{-1 / 2} \text {, }
$$

where $B_{0}^{(1)}$ is given in (3.20), it follows immediately from (5.1), (5.21) and (5.30) that

$$
\text { (5.35) } \begin{aligned}
S_{n}^{*}= & B_{0}^{(1)}+\frac{\sqrt{2}}{\pi^{3 / 2}} N^{1 / 2}\left(\int_{0}^{\pi / 2}-\int_{0}^{j_{1,1} / N}-\int_{j_{1, n} / N}^{\pi / 2}\right) g(\theta) \theta^{-1 / 2} d \theta \\
& +\frac{1}{2 \sqrt{\pi}} N^{-1 / 2}+\varepsilon_{16}+\varepsilon_{20}+\varepsilon_{24} .
\end{aligned}
$$


Recall from (3.22) that

Hence

$$
|g(\theta)-1| \leq \frac{1}{2}\left|g^{\prime \prime}\left(\frac{5 \pi}{4 N}\right)\right| \theta^{2}, \quad 0 \leq \theta \leq \frac{5 \pi}{4 N} .
$$

$$
\int_{0}^{j_{11} / N} g(\theta) \theta^{-1 / 2} d \theta=N^{-1 / 2} \int_{0}^{j_{1,1}} x^{-1 / 2} d x+\varepsilon_{25}
$$

where

$$
\left|\varepsilon_{25}\right| \leq 0.4791 N^{-5 / 2} \text { for } N \geq 50 .
$$

Coupling (5.35) and (5.36), we obtain (3.19) with

$$
\varepsilon_{9}=\varepsilon_{16}+\varepsilon_{20}+\varepsilon_{24}-\frac{\sqrt{2}}{\pi^{3 / 2}} N^{1 / 2} \varepsilon_{25} .
$$

This completes the proof of Lemma 5.

6. Calculation of $L_{n}^{(2)}$. By Corollary $1^{\prime}$,

$$
L_{n}^{(2)}=\frac{1}{\sqrt{2}} \int_{0}^{\pi / 2}\left(\frac{\hat{f}(\theta)}{\hat{f}^{\prime}(\theta)}\right)^{1 / 2}\left(\tan \frac{\theta}{2}\right)^{1 / 2}\left|J_{0}(\hat{f}(\theta))\right| d \theta+\hat{\varepsilon}_{5}
$$

where

$$
\left|\hat{\varepsilon}_{5}\right| \leq \int_{0}^{\pi / 2}\left(\frac{\hat{f}(\theta)}{\hat{f}^{\prime}(\theta)}\right)^{1 / 2}\left(\tan \frac{\theta}{2}\right)^{1 / 2}|\hat{I}| d \theta .
$$

Since $0 \leq \tan (\theta / 2) \leq 1$ and $0 \leq \hat{f}(\theta) \leq N \theta$ for $\theta \in[0, \pi / 2]$, it can be shown that

$$
\left|\hat{\varepsilon}_{5}\right| \leq 0.0141 N^{-5 / 2} \text { for } N \geq 50 .
$$

Here we wish to point out that the function $\hat{f}_{1}(\theta)=3 \tan (\theta / 2)-(2 / \theta-$ $\cot (\theta / 2))$ in (2.15) and all its derivatives are positive and increasing in $[0, \pi / 2]$. Furthermore, the functions $\hat{f}(\theta)=N \theta-(1 / 16 N) \hat{f}_{1}(\theta)$ and $(1 / \theta) \hat{f}_{1}(\theta)$ are also increasing in that interval. Following (3.9), we split the interval of integration in (6.1) at $\hat{\tau}_{k}$, the root of the equation $\hat{f}(\theta)=j_{0, k}$. (Note that $j_{0, k} / N<\hat{\tau}_{k}<j_{0, k+1} / N$.) Thus

$$
L_{n}^{(2)}=\frac{1}{\sqrt{2}}\left[\hat{I}_{0}+\sum_{k=1}^{m-1}(-1)^{k} \hat{I}_{k}+(-1)^{\bar{m}} \hat{I}_{m}\right]+\hat{\varepsilon}_{5},
$$

where

$$
\begin{aligned}
\hat{I}_{k}=\int_{\hat{\tau}_{k}}^{\hat{\tau}_{k+1}}\left(\frac{\hat{f}(\theta)}{\hat{f}^{\prime}(\theta)}\right)^{1 / 2}\left(\tan \frac{\theta}{2}\right)^{1 / 2} J_{0}(\hat{f}(\theta)) d \theta \\
k=0,1, \ldots, \bar{m}-1,
\end{aligned}
$$


and

(6.6) $\hat{I}_{\bar{m}}=\int_{\hat{\tau}_{m}}^{\pi / 2}\left(\frac{\hat{f}(\theta)}{\hat{f}^{\prime}(\theta)}\right)^{1 / 2}\left(\tan \frac{\theta}{2}\right)^{1 / 2} J_{0}(\hat{f}(\theta)) d \theta, \quad \bar{m}=n-\bar{n}$.

For convenience, we have set $\hat{\tau}_{0} \equiv 0$.

In view of the identity $x J_{0}(x)=\left[x J_{1}(x)\right]^{\prime}$, the integral $\hat{I}_{k}$ can be expressed as

$$
\hat{I}_{k}=\int_{\hat{\tau}_{k}}^{\hat{\tau}_{k+1}} \hat{G}(\theta) d\left[\hat{f}(\theta) J_{1}(\hat{f}(\theta))\right]
$$

where

$$
\hat{G}(\theta)=\frac{1}{\sqrt{2}}\left(\frac{\theta}{\hat{f}(\theta)}\right)^{1 / 2}\left[\hat{f}^{\prime}(\theta)\right]^{-3 / 2} \hat{g}(\theta)
$$

and

$$
\hat{g}(\theta)=\left(\frac{2}{\theta} \tan \frac{\theta}{2}\right)^{1 / 2} .
$$

Note that the function $\hat{g}(\theta)$ is increasing in $[0, \pi / 2]$. The result corresponding to $(4.5)$ is

$$
\hat{G}(\theta)=\frac{1}{\sqrt{2}} N^{-2} \hat{g}(\theta)+\hat{\varepsilon}_{12},
$$

where

$$
\left|\hat{\varepsilon}_{12}\right| \leq 0.2650 N^{-4} \text { for } N \geq 50 .
$$

Inserting (6.10) in (6.7) and applying an integration by parts, we obtain, upon summing up,

$$
\begin{aligned}
\sum_{k=1}^{m-1}(-1)^{k} \hat{I}_{k}= & \frac{1}{\sqrt{2}} N^{-2} \hat{S}_{n}^{*} \\
& +\frac{1}{\sqrt{2}} N^{-2}\left[\hat{g}\left(\hat{\tau}_{1}\right) \hat{M}_{1}-\hat{g}\left(\hat{\tau}_{\bar{m}}\right) \hat{M}_{\bar{m}}\right]+\hat{\varepsilon}_{13}+\hat{\varepsilon}_{14},
\end{aligned}
$$

where $\hat{M}_{k}$ is as given in (1.12) and

$$
\hat{S}_{n}^{*}=\sum_{k=2}^{m} \hat{g}\left(\hat{\tau}_{k}\right) \hat{M}_{k}
$$

The error terms $\hat{\varepsilon}_{13}$ and $\hat{\varepsilon}_{14}$ correspond to those given in (4.9) and (4.12), respectively. It can be shown, as in $\S 4$, that

$$
\left|\hat{\varepsilon}_{13}\right| \leq 0.1830 N^{-5 / 2}
$$


and

$$
\left|\hat{\varepsilon}_{14}\right| \leq 0.9712 N^{-5 / 2}
$$

for $n \geq 50$. The analogues of (3.15) and (3.16) are

$$
\hat{I}_{0}=\frac{1}{\sqrt{2}} N^{-2} \int_{0}^{j_{0.1}} y J_{0}(y) d y+\hat{\varepsilon}_{7}=\frac{1}{\sqrt{2}} N^{-2} \hat{M}_{1}+\hat{\varepsilon}_{7}
$$

and

$$
\begin{aligned}
& (-1)^{\bar{m}} \hat{I}_{\bar{m}} \\
& =\frac{1}{\sqrt{2}} N^{-2}\left[\hat{g}\left(\hat{\tau}_{\bar{m}}\right) \hat{M}_{\bar{m}}+(-1)^{\bar{m}} \hat{g}\left(\frac{\pi}{2}\right) \hat{f}\left(\frac{\pi}{2}\right) J_{1}\left(\hat{f}\left(\frac{\pi}{2}\right)\right)\right] \\
& \quad+\hat{\varepsilon}_{8}
\end{aligned}
$$

where

$$
\left|\hat{\varepsilon}_{7}\right| \leq 0.0019 N^{-5 / 2} \text { and }\left|\hat{\varepsilon}_{8}\right| \leq 0.1927 N^{-5 / 2}
$$

for $N \geq 50$. In a manner similar to Lemma 5 (cf. (3.19)), it can also be proved that

$$
\begin{aligned}
\hat{S}_{n}^{*}= & D_{0}-\hat{M}_{1}+\frac{\sqrt{2}}{\pi^{3 / 2}} N^{3 / 2}\left(\int_{0}^{\pi / 2}-\int_{j_{0, m} / N}^{\pi / 2}\right) \hat{g}(\theta) \theta^{1 / 2} d \theta \\
& +\frac{1}{\sqrt{\pi}} N^{1 / 2}+\hat{\varepsilon}_{9}
\end{aligned}
$$

where

$$
\left|\hat{\varepsilon}_{9}\right| \leq 3.489 N^{-1 / 2}
$$

and $D_{0}$ has the same meaning as given in (2.50). The final asymptotic formula for $L_{n}^{(2)}$, given in (3.30), is obtained by combining the results in (6.12), (6.16), (6.17) and (6.19).

Observe that the coefficient in the approximation (6.10) for $\hat{G}(\theta)$ is $1 / \sqrt{2}$, whereas the corresponding coefficient for $G(\theta)$ in (4.5) is $\sqrt{2}$. Thus, the approximations differ by a factor of two. Comparing equations (3.12), (3.15) and (3.16) with the corresponding equations (6.12), (6.16) and (6.17), one notices that this difference carries through the calculations of $L_{n}^{(1)}$ and $L_{n}^{(2)}$. This explains why the error $E_{1}$ in (3.28) is approximately twice as large as that in (3.30).

7. The sum of $L_{n}^{(1)}$ and $L_{n}^{(2)}$. From (3.28) and (3.30), we have

$$
L_{n}=I_{1}^{*}+I_{2}^{*}+I_{3}^{*}+B_{0}+\frac{2}{\sqrt{\pi}} N^{-1 / 2}+D_{0} N^{-1}+E_{1}+E_{2},
$$


where

$$
\begin{aligned}
I_{1}^{*}=\frac{2}{\pi^{3 / 2}} N^{1 / 2}\left[\int_{0}^{\pi / 2}\right. & \left(\sin \frac{\theta}{2}\right)^{-1 / 2}\left(\cos \frac{\theta}{2}\right)^{1 / 2} d \theta \\
& \left.+\int_{0}^{\pi / 2}\left(\sin \frac{\theta}{2}\right)^{1 / 2}\left(\cos \frac{\theta}{2}\right)^{-1 / 2} d \theta\right]
\end{aligned}
$$

(7.3) $I_{2}^{*}=-\frac{2}{\pi^{3 / 2}} N^{1 / 2}\left[\int_{j_{1, n} / N}^{\pi / 2}\left(\sin \frac{\theta}{2}\right)^{-1 / 2}\left(\cos \frac{\theta}{2}\right)^{1 / 2} d \theta\right.$

$$
\left.+\int_{j_{0} / N}^{\pi / 2}\left(\sin \frac{\theta}{2}\right)^{1 / 2}\left(\cos \frac{\theta}{2}\right)^{-1 / 2} d \theta\right]
$$

and

$$
\begin{aligned}
I_{3}^{*}= & (-1)^{n+1} g\left(\frac{\pi}{2}\right) J_{0}\left(f\left(\frac{\pi}{2}\right)\right) \\
& +\frac{(-1)^{m}}{2} N^{-1} \hat{g}\left(\frac{\pi}{2}\right) \hat{f}\left(\frac{\pi}{2}\right) J_{1}\left(\hat{f}\left(\frac{\pi}{2}\right)\right) .
\end{aligned}
$$

By letting $\theta=\pi-\phi$ in the second integral in (7.2), the two integrals there can be combined into the single integral

$$
\int_{0}^{\pi}\left(\sin \frac{\theta}{2}\right)^{-1 / 2}\left(\cos \frac{\theta}{2}\right)^{1 / 2} d \theta=\sqrt{2} \cdot \pi
$$

Thus, since $N=n+1,(7.2)$ can be expressed as

$$
I_{1}^{*}=\frac{2 \sqrt{2}}{\sqrt{\pi}} n^{1 / 2}+\sqrt{\frac{2}{\pi}} n^{-1 / 2}+\varepsilon_{1}^{*},
$$

where $\left|\varepsilon_{1}^{*}\right|=0.1995 n^{-3 / 2}$. By the same argument, the two integrals in (7.3) can be combined into the single integral so that

$$
I_{2}^{*}=-\frac{2}{\pi^{3 / 2}} N^{1 / 2} \int_{j_{1,} / N}^{\pi-j_{0, m} / N}\left(\cot \frac{\theta}{2}\right)^{1 / 2} d \theta .
$$

Since both limits of integration tend to $\pi / 2$ as $n \rightarrow \infty$, we expand the integrand $h(\theta)=(\cot (\theta / 2))^{1 / 2}$ at $\theta=\pi / 2$ :

$$
\left(\cot \frac{\theta}{2}\right)^{1 / 2}=1+h^{\prime}(\eta)\left(\theta-\frac{\pi}{2}\right) \text {. }
$$

$\eta$ being between $\theta$ and $\pi / 2$. Note that both $j_{1, \tilde{n}} / N$ and $j_{0, \tilde{m}} / N$ are less than $\pi / 2$, and hence that the upper limit in (7.7) is indeed greater 
than the lower limit of integration. Inserting (7.8) in (7.7), we obtain by the argument following (5.28)

$$
I_{2}^{*}=-\frac{2}{\pi^{3 / 2}} N^{1 / 2}\left[\pi-\frac{j_{0, \bar{m}}}{N}-\frac{j_{1, \bar{n}}}{N}\right]+\varepsilon_{2}^{*}
$$

with $\left|\varepsilon_{2}^{*}\right| \leq 0.7555 N^{-3 / 2}$. Here use has also been made of the inequality $\pi / 2-\pi / 4 N<j_{0, m} / N$. From Lemmas 2 and $2^{\prime}$, it follows that

$$
I_{2}^{*}=-\frac{2}{\sqrt{\pi}} N^{-1 / 2}+\varepsilon_{3}^{*},
$$

where $\left|\varepsilon_{3}^{*}\right| \leq 0.7933 n^{-3 / 2}$.

To approximate $I_{3}^{*}$, we first recall the asymptotic approximation

$$
J_{0}(x)=\sqrt{\frac{2}{\pi x}} \cos \left(x-\frac{\pi}{4}\right)+\varepsilon_{4}^{*}
$$

where

$$
\left|\varepsilon_{4}^{*}\right| \leq \sqrt{\frac{2}{\pi}} \cdot \frac{1}{8} x^{-3 / 2}, \quad x>0
$$

Next we observe that if $n$ is even, then

$$
\begin{aligned}
\cos \left[f\left(\frac{\pi}{2}\right)-\frac{\pi}{4}\right]=\frac{1}{\sqrt{2}}\left\{(-1)^{(n / 2)+1}\right. & \sin \left[\frac{1}{4 N}\left(1-\frac{3}{\pi}\right)\right] \\
& \left.+(-1)^{n / 2} \cos \left[\frac{1}{4 N}\left(1-\frac{3}{\pi}\right)\right]\right\},
\end{aligned}
$$

and that if $n$ is odd, then

$$
\begin{aligned}
\cos \left[f\left(\frac{\pi}{2}\right)-\frac{\pi}{4}\right]=\frac{1}{\sqrt{2}}\left\{(-1)^{(n+1) / 2} \cos \left[\frac{1}{4 N}\left(1-\frac{3}{\pi}\right)\right]\right. \\
\left.+(-1)^{(n+1) / 2} \sin \left[\frac{1}{4 N}\left(1-\frac{3}{\pi}\right)\right]\right\} .
\end{aligned}
$$

The last two equations can be combined to give

$$
\cos \left[f\left(\frac{\pi}{2}\right)-\frac{\pi}{4}\right]=\frac{1}{\sqrt{2}} \begin{cases}(-1)^{n / 2}+\varepsilon_{5}^{*}, & n \text { is even, } \\ (-1)^{(n+1) / 2}+\varepsilon_{5}^{*}, & n \text { is odd }\end{cases}
$$

with $\left|\varepsilon_{5}^{*}\right| \leq 0.0080 N^{-1}$. Since $g(\pi / 2)=\sqrt{\pi} / 2$ and

$$
\left[f\left(\frac{\pi}{2}\right)\right]^{-1 / 2}=\sqrt{\frac{2}{\pi}} N^{-1 / 2}+\varepsilon_{6}^{*}
$$

with $\left|\varepsilon_{6}^{*}\right| \leq 0.0029 N^{-5 / 2}$, a combination of these results gives

$$
(-1)^{n+1} g\left(\frac{\pi}{2}\right) J_{0}\left(f\left(\frac{\pi}{2}\right)\right)=(-1)^{n+1} \frac{1}{\sqrt{2 \pi}} N^{-1 / 2}+\varepsilon_{7}^{*},
$$


where $\left|\varepsilon_{7}^{*}\right| \leq 0.0495 N^{-3 / 2}$. In a similar manner, one can show that

$$
\frac{(-1)^{m}}{2} N^{-1} \hat{g}\left(\frac{\pi}{2}\right) \hat{f}\left(\frac{\pi}{2}\right) J_{1}\left(\hat{f}\left(\frac{\pi}{2}\right)\right)=\frac{(-1)^{n}}{\sqrt{2 \pi}} N^{-1 / 2}+\varepsilon_{8}^{*},
$$

where $\left|\varepsilon_{8}^{*}\right| \leq 0.2314 N^{-3 / 2}$. Note that the leading terms in (7.14) and (7.15) differ only by a minus sign, and hence that

$$
I_{3}^{*}=\varepsilon_{7}^{*}+\varepsilon_{8}^{*}
$$

By (2.51), we also have

$$
D_{0} N^{-1}=D_{0} n^{-1}+\varepsilon_{9}^{*}
$$

where $\left|\varepsilon_{9}^{*}\right| \leq 0.3849 n^{-3 / 2}$. The final result (1.10) now follows upon adding (7.6), (7.10), (7.16) and (7.17) together. The error term $\varepsilon(n)$ in (1.10) is given by

$$
\varepsilon(n)=\varepsilon_{1}^{*}+\varepsilon_{3}^{*}+\varepsilon_{7}^{*}+\varepsilon_{8}^{*}+\varepsilon_{9}^{*}+E_{1}+E_{2},
$$

and hence satisfies the estimate (1.14).

8. Conclusion. In this paper we have found an error bound for a four-term asymptotic expansion of the Lebesgue constants for Legendre series. From this we have also shown that these constants are indeed monotonically increasing, a conjecture of Szegö which dates back to 1926. The development of error theories for asymptotic approximations has been advocated by F. W. J. Olver [11] for some time. The present paper is another demonstration of the usefulness of a well-constructed error bound.

Although Szegö's conjecture is now proved, the present approach is far too complicated. A more satisfactory approach would be to search for an alternative expression for the Lebesgue constants from which the monotonicity of these constants is evident. This is the approach which Szegö had used to show that the sequence of differences of the Lebesgue constants for trigonometric Fourier series is completely monotonic. We shall, however, leave this problem to the experts in orthogonal polynomials.

Acknowledgment. We would like to express our sincere thanks to Professor L. Gatteschi for presenting us a copy of Reference [2] prior to its publication, and to Mr. Tom Lang for carrying out the numerical computations of $L_{n}(1 \leq n \leq 50)$ given in $\S 3$. We would also like to thank Professor L. Lorch for providing us with some historical background to the problem. The possibility of using a Cherry-type 
approximation (2.7) was suggested to us by Professor F. W. J. Olver, to whom we wish to express our gratitude.

\section{REFERENCES}

[1] R. Askey, Ed., The Collected Papers of Gabor Szegö, Vol. 1, Birkhäuser, Cambridge, MA, 1982.

[2] P. Baratella and L. Gatteschi, The bounds for the error term of an asymptotic approximation of Jacobi polynomials, Proceedings of the 2nd International Symposium on Orthogonal Polynomials and Their Applications, Segovia/Spain 1986, F. Marcellan, Ed.

[3] T. M. Cherry, Uniform asymptotic formulae for functions with transition points, Trans. Amer. Math. Soc., 68 (1950), 224-257.

[4] L. Fejer, Lebesguesche Konstanten und divergente Fourierreihen, J. Reine Angew. Math., 138 (1910), 22-53.

[5] C. L. Frenzen and R. Wong, $A$ uniform asymptotic expansion of the Jacobi polynomials with error bounds, Canad. J. Math., 37 (1985), 979-1007.

[6] Asymptotic expansions of the Lebesgue constants for Jacobi series, Pacific J. Math., 122 (1986), 391-415.

[7] T. H. Gronwall, Über die Lebesgueschen Konstanten bei den Fourierschen Reihen, Math. Ann., 72 (1912), 244-261.

[8] _ Über die Laplacesche Reihe, Math. Ann., 74 (1913), 213-270.

[9] H. W. Hethcote, Asymptotic approximations with error bounds for zeros of Airy and cylindrical functions, Doctoral thesis, University of Michigan, Ann Arbor, 1968.

[10] L. Lorch, The Lebesgue constants for Jacobi series, II, Amer. J. Math., 81 (1959), 875-888.

[11] F. W. J. Olver, Asymptotic approximations and error bounds, SIAM Review, 22 (1980), 188-203.

[12] G. Szegö, Über die Lebesgueschen Konstanten bei den Fourierschen Reihen, Math. Z., 9 (1921), 163-166.

[13] A Lebesgue-féle âllandók elméletéhez, in The Collected Papers of Gabor Szegö, Vol. 1, pp. 777-783, R. Askey, Ed., Birkhäuser, Cambridge, MA, 1982.

[14] _ Über einige asymptotische Entwicklungen der Legendreschen Functionen, Proc. London Math. Soc., (2), 36 (1932), 427-450.

[15] _ـ Über eine von Herrn S. Berstein herrührende Abschätzung der Legendreschen Polynome, Math. Ann., 108 (1933), 360-369.

[16] _ Orthogonal Polynomials, Colloquium Publications, Vol. 23, 3rd ed., Amer. Math. Soc., Providence, RI, 1967.

[17] G. N. Watson, A Treatise on the Theory of Bessel Functions (2nd ed.), Cambridge University Press, London and New York, 1944.

[18] A. Zygmund, Trigonometric Series, Warsaw, 1935.

Received May 11, 1987. This research was partially supported by the Natural Sciences and Engineering Research Council of Canada under grant A7359.

\section{TSINGHUA UNIVERSITY \\ BeIJIN, China}

AND

UNIVERSITY OF MANITOBA

WinNipeg, MaNitoba, CaNada, R3T 2N2 